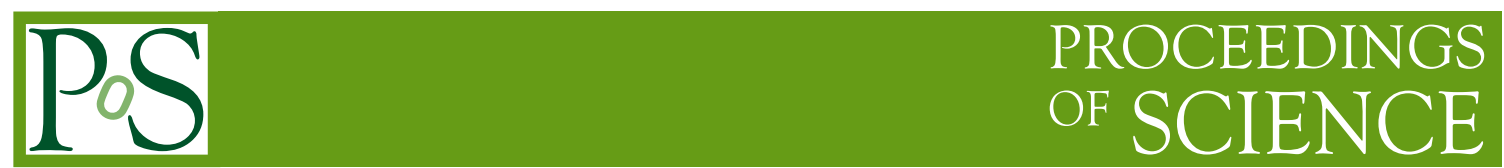

\title{
Performance of the Belle II Silicon Vertex Detector
}

\section{Christoph Schwanda*}

Institute of High Energy Physics, Austrian Academy of Sciences, 1050 Vienna, Austria

E-mail: Christoph. Schwanda@oeaw.ac.at

K. Adamczyk ${ }^{w}$, H. Aihara ${ }^{t}$, T. Aziz ${ }^{k}$, S. Bacher ${ }^{w}$, S. Bahinipati ${ }^{g}$, G. Batignani ${ }^{l, m}$,

J. Baudot ${ }^{f}$, P. K. Behera ${ }^{h}$,S. Bettarini ${ }^{l, m}$,T. Bilka ${ }^{e}$, A. Bozek ${ }^{w}$, F. Buchsteiner ${ }^{b}$,

G. Casarosa ${ }^{l, m}$, D. Červenkov ${ }^{e}$, Y. Q. Chen ${ }^{d}$, L. Corona ${ }^{l, m}$, T. Czank ${ }^{r}$, S. B. Das ${ }^{i}$,

N. Dash ${ }^{h}$, G. de Marino ${ }^{l, m}$, Z. Doležal ${ }^{e}$, G. Dujany ${ }^{f}$, F. Forti $^{l, m}$, M. Friedl $^{b}$, E. Ganiev ${ }^{o, p}$,

B. Gobbo ${ }^{p}$, S. Halder ${ }^{k}$, K. Hara $^{u, q}$, S. Hazra ${ }^{k}$, T. Higuchi $^{r}$, C. Irmler ${ }^{b}$, A. Ishikawa ${ }^{u, q}$,

H. B. Jeon ${ }^{v}$, C. W. Joo ${ }^{r}$, M. Kaleta ${ }^{w}$, A. B. Kaliyar ${ }^{k}$, J. Kandra ${ }^{e}$, K. H. Kang ${ }^{v}$,

P. Kapusta ${ }^{w}$, P. Kodyš ${ }^{e}$, T. Kohriki ${ }^{u}$, M. Kumar ${ }^{i}$, R. Kumar ${ }^{j}$, P. Kvasnička ${ }^{e}$, C. La

Licata $^{r}$, K. Lalwani ${ }^{i}$, L. Lanceri ${ }^{o, p}$, S. C. Lee ${ }^{v}$, Y. B. Li ${ }^{c}$, J. Libby ${ }^{h}$, T. Lueck ${ }^{l, m, n}$,

S. Maity ${ }^{g}$, S. N. Mayekar ${ }^{k}$, G. B. Mohanty ${ }^{k}$, J. A. Mora Grimaldo ${ }^{t}$, T. Morii ${ }^{r}$,

K. R. Nakamura ${ }^{u, q}$, Z. Natkaniec ${ }^{w}$, Y. Onuki ${ }^{t}$, W. Ostrowicz ${ }^{w}$, A. Paladino ${ }^{l, m}$,

E. Paoloni ${ }^{l}, m$, H. Park ${ }^{v}$, K. K. Rao ${ }^{k}$, I. Ripp-Baudot ${ }^{f}$, G. Rizzo ${ }^{l, m}$, N. Rout ${ }^{h}$, D. Sahoo ${ }^{k}$,

N. Sato ${ }^{u}$, J. Suzuki ${ }^{u}$, S. Tanaka ${ }^{u, q}$, H. Tanigawa $^{t}$, R. Thalmeier ${ }^{b}$, T. Tsuboyama ${ }^{u, q}$,

Y. Uematsu ${ }^{t}$, O. Verbycka ${ }^{w}$, L. Vitale ${ }^{o, p}, \mathbf{K}$. Wan $^{t}$, S. Watanuki ${ }^{s}$, J. Webb ${ }^{a}$,

J. Wiechczynski ${ }^{m}$, H. Yin ${ }^{b}$, L. Zani ${ }^{l, m}$, T. Zhang ${ }^{t}$. 
School of Physics, University of Melbourne, Melbourne, Victoria 3010, Australia

${ }^{b}$ Institute of High Energy Physics, Austrian Academy of Sciences, 1050 Vienna, Austria

${ }^{c}$ Peking University, Department of Technical Physics, Beijing 100871, China

${ }^{d}$ University of Science and Technology of China, Department of Modern Physics, Hefei 230026, China

${ }^{e}$ Faculty of Mathematics and Physics, Charles University, 12116 Prague, Czech Republic

${ }^{f}$ IPHC, UMR 7178, Université de Strasbourg, CNRS, 67037 Strasbourg, France

${ }^{g}$ Indian Institute of Technology Bhubaneswar, Satya Nagar, India

${ }^{h}$ Indian Institute of Technology Madras, Chennai 600036, India

${ }^{i}$ Malaviya National Institute of Technology, Jaipur 302017, India

${ }^{j}$ Punjab Agricultural University, Ludhiana 141004, India

${ }^{k}$ Tata Institute of Fundamental Research, Mumbai 400005, India

${ }^{l}$ Dipartimento di Fisica, Università di Pisa, I-56127 Pisa, Italy

${ }^{m}$ INFN Sezione di Pisa, I-56127 Pisa, Italy

${ }^{n}$ Presently at Ludwig Maximilian University of Munich, 80539 Munich, Germany

${ }^{o}$ Dipartimento di Fisica, Università di Trieste, I-34127 Trieste, Italy

${ }^{p}$ INFN Sezione di Trieste, I-34127 Trieste, Italy

${ }^{q}$ The Graduate University for Advanced Studies (SOKENDAI), Hayama 240-0193, Japan

${ }^{r}$ Kavli Institute for the Physics and Mathematics of the Universe (WPI), University of Tokyo, Kashiwa 277-8583, Japan

${ }^{s}$ Department of Physics, Tohoku University, Sendai 980-8578, Japan

${ }^{t}$ Department of Physics, University of Tokyo, Tokyo 113-0033, Japan

${ }^{u}$ High Energy Accelerator Research Organization (KEK), Tsukuba 305-0801, Japan, ${ }^{A}$ deceased

${ }^{v}$ Department of Physics, Kyungpook National University, Daegu 702-701, Korea

${ }^{w}$ H. Niewodniczanski Institute of Nuclear Physics, Krakow 31-342, Poland

The Belle II experiment at the SuperKEKB collider of KEK (Japan) will accumulate $50 \mathrm{ab}^{-1}$ of $e^{+} e^{-}$collision data at an unprecedented instantaneous luminosity of $8 \times 10^{35} \mathrm{~cm}^{-2} \mathrm{~s}^{-1}$, about 40 times larger than its predecessor Belle. The Belle II vertex detector (VXD) provides inner tracking, impact parameter measurement and decay vertex reconstruction for the Belle II detector and consists of two layers of DEPFET-based pixels (PXD) and four layers of double sided silicon strip detectors (SVD), the latter being the focus of this paper. We review the design of the SVD and its assembly and commissioning, and report preliminary results obtained with SVD during the first physics run of Belle II from March to June 2019.

The 28th International Workshop on Vertex Detectors - Vertex2019

13-18 October, 2019

Lopud, Croatia

${ }^{*}$ Speaker. 


\section{Introduction}

The Belle II experiment [1] at the SuperKEKB collider [2] is the result of the upgrade of the $B$ factory facility at the KEK laboratory (Tsukuba, Japan), which was performed throughout the years 2011 to 2018. SuperKEKB collides a $4 \mathrm{GeV}$ positron beam (LER) with a $7 \mathrm{GeV}$ electron beam (HER), thus allowing for the on-threshold production of a $B$ meson pair at the energy of the $\Upsilon(4 S)$ resonance $(10.58 \mathrm{GeV})$. The machine aims for an instantaneous luminosity of $8 \times 10^{35} \mathrm{~cm}^{-2} \mathrm{~s}^{-1}$, about a factor of 40 higher than at the previous $B$ factory facility [3].

The experiment, located in the single interaction region of the LER and HER beams, performs precision flavor physics in order to confirm or reject anomalies observed in the previous generation of flavor physics experiments. The challenge for the Belle II detector is thus to record $e^{+} e^{-}$collision data with high quality despite the harsh high luminosity environment characterized by increased machine and luminosity-dependent backgrounds. Another challenge is to maintain the same relative precision of $B$ decay vertex separation despite the reduced relativistic boost of the $\Upsilon(4 S)(\beta \gamma=0.28$ compared to 0.425 at the previous machine $)$.

In this paper, we review the design of the Silicon Vertex Detector (SVD), which corresponds to the outer part of the Belle II Vertex Detector (VXD). We briefly describe the SVD assembly and first operation during SVD stand-alone commissioning. The main part of this paper covers the results and the operational experience obtained with SVD during its first physics run in spring 2019 (March to June 2019).

\section{SVD design}

The Belle II VXD consists of two layers of DEPFET-based pixel sensors (PXD) [4] and four layers of double-sided silicon strip sensors (SVD), arranged cylindrically around the beam pipe. It operates in the $1.5 \mathrm{~T}$ magnetic field of the Belle II solenoid and provides inner tracking, impact parameter measurement and decay vertex reconstruction for the Belle II detector. Fig. 1 shows a picture of one completed VXD half.

Defining the axis of the Belle II solenoid as the $z$ axis, the SVD covers all azimuth angles $\phi$ and a polar angle $\theta$ region from $17^{\circ}$ to $150^{\circ}$. Moving from the in- to the outside, the SVD layers (referred to as layers 3, 4, 5 and 6) are located at radii $R$ (measured from the $z$ axis) of $39 \mathrm{~mm}$, $80 \mathrm{~mm}, 105 \mathrm{~mm}$ and $135 \mathrm{~mm}$. Layers 3, 4, 5 and 6 consist of 7, 10, 12 and 16 longitudinal modules each, respectively, called ladders, which are mounted in parallel to the beam pipe on the end-ring structure.

Layer 3 modules consist of two double-sided silicon strip detectors (DSSDs) with $320 \mu \mathrm{m}$ thickness and 768 readout strips on both the $n$ - and $p$-sides, oriented in the $R \phi$ and $z$ directions, respectively. The readout pitch is $160 \mu \mathrm{m}$ on the $n$ - and $50 \mu \mathrm{m}$ on the $p$-side. The front-end readout ASIC is the APV25 chip, originally developed for the CMS experiment [5]. With its shaping time of $50 \mathrm{~ns}$, this chip is fast enough to keep the occupancy in layer 3 within acceptable limits for particle track reconstruction. The APV25 has 128 input channels and can tolerate a radiation dose beyond $100 \mathrm{Mrad}$. For the L3 ladders, the front-end readout chips are placed on hybrid boards at the near and far end of the modules, outside of the detector acceptance window. Each chip dissipates 


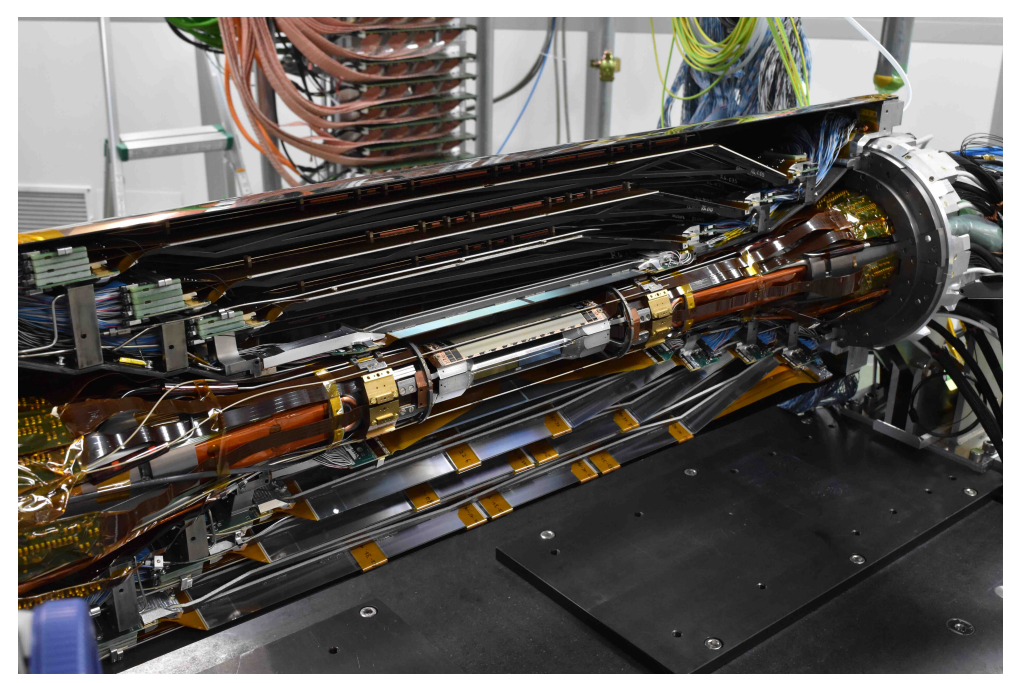

Figure 1: One half of the Belle II vertex detector (VXD).

about $0.4 \mathrm{~W}$ and $\mathrm{L} 3$ cooling is provided by a two-phase $\mathrm{CO}_{2}$ cooling system using channels inside the end-ring structure.

The ladders for L4, L5 and L6 use larger, rectangular DSSDs ( $n$-side: 512 readout strips with $240 \mu \mathrm{m}$ readout pitch; $p$-side: 768 readout strips with $75 \mu \mathrm{m}$ readout pitch; thickness: $320 \mu \mathrm{m}$ ) and a trapezoidal DSSD in the forward $\theta$ region ( $n$-side: 512 readout strips with $240 \mu$ m readout pitch; $p$-side: 768 readout strips with a readout pitch varying between 50 and $75 \mu \mathrm{m}$; thickness: $300 \mu \mathrm{m}$ ). The forward sensors are mounted at an angle to the beam pipe giving a lamp-shade geometry to the SVD. Only the first and the last sensors of a ladder are read out from the side (as in L3) while the sensors in the middle use the origami readout: Here, the APV25 chips (thinned down to $100 \mu \mathrm{m}$ ) are placed on the DSSD $n$-side (within the detector acceptance) and DSSD strips on both sides are connected by flexible printed circuits. The pitch adapters for the $p$-side strips are wrapped around the edge of the origami sensor. $\mathrm{CO}_{2}$ cooling is brought to the readout ASICs lined up on the sensor $n$-side by a thin stainless steel pipe.

In summary, the SVD has an active DSSD area of about $1.1 \mathrm{~m}^{2}$ and is read out by atal of 1748 APV25 chips. The material budget per DSSD layer corresponds to $0.7 \%$ of radiation length. The detector has been designed for an integrated radiation dose of $10 \mathrm{Mrad}$, which should be well within the limit of the experiment lifetime.

\section{SVD assembly and commissioning}

While SVD ladders were assembled by five groups (Pisa, Melbourne, TIFR Mumbai, HEPHY Vienna and Kavli IPMU Kashiwa) at their respective facilities, the ladder mount and SVD assembly were performed on site at KEK. The SVD was assembled in two half shells, which were subsequently mounted onto the beam pipe after the PXD assembly. Finally, the entire VXD was inserted into the central part of the Belle II detector. 
The first half shell (called $+x$ by reference to the standard Belle II coordinate frame) was completed in January 2018 and the second $(-x)$ followed in July 2018. Between July and September 2018 the two half shells were operated in a dry box in the Tsukuba experimental hall (close to the experiment) and cosmic data and noise runs were taken for detector alignment and calibration as well as to gain some operational experience with the system. In total about 30 million cosmic events were recorded with both SVD halves during this SVD stand-alone commissioning phase. The operation of all sensors and the stability of calibration parameters have been confirmed.

In October 2018, the SVD half shells were combined with the PXD and the full VXD was installed in November 2018. In general, despite the mechanical and electrical complexity of these final assembly steps, we did not experience any major trouble at this stage but we had to deal with occasional connection troubles (such as mechanical damage to the cables connecting the APV25 hybrid boards), which could be spotted and repaired quickly due to repeated electrical sanity tests.

\section{Spring 2019 run}

Belle II started its first physics run with the VXD detector installed in March 2019 and integrated a luminosity of about $6.5 \mathrm{fb}^{-1}$ on the $\Upsilon(4 S)$ resonance until the end of June 2019. This figure is below original plans partly due a fire incident close to the accelerator facility which caused a month-long halt of the machine in April 2019. SVD operation throughout this run has been stable and no major issue was encountered. As explained in more detail in Sect. 6, the efficiency for SVD strip clusters has been around $99 \%$ in all layers and on both the $n$ - and the $p$-sides. The occupancy, i.e., the average percentage of strips with a signal over threshold at any trigger, is dominated in this run by hits from machine backgrounds. It is highest in L3 and has been below $0.3 \%$, which is still an order of magnitude below the limit for good tracking performance.

The following minor issues were encountered:

- One front-end readout ASIC caused frequent errors during physics runs due to an unstable connection and has been disabled on March 27. This corresponds to about $1.2 \%$ of the L3 $n$-side acceptance. The location of the connection issue was inside the Belle II detector but actually outside of the SVD and the function of the chip could be restored during summer 2019 shutdown.

- Two sensors showed a leakage current far above the typical 1-2 $\mu \mathrm{A}$ at the nominal $100 \mathrm{~V}$ bias voltage. This did not affect the physics performance of the corresponding sensors, i.e., the signal-to-noise ratio of the concerned strips has been at normal levels. While for one sensor this behavior was erratic and not clearly understood, for the second sensor the current was clearly beam related: During operation with beam and bias voltage on, the current increased at a rate of about $4 \mu \mathrm{A} /$ day. However, during operation with beam and high voltage off, the effect could be reverted and the sensor current returned to normal. We believe that this effect is due to static charge accumulation on the sensor surface, causing a localized higher electric field on the junction side, close to some defects, and producing an increased sensor current by impact ionization. We could in fact mitigate this effect and stabilize the sensor current modifying the high voltage configuration such that the electric field in air (between ladders of different layers) was reduced. 
- We experienced a few failures of low voltage supply boards.

- Finally, the SVD was also affected by the SuperKEKB radiation accidents of May 28, June 6 and June 9. During these events a final focusing QCS magnet quenched and part of the beam entered the detector before it could be safely dumped. Luckily no significant damage was caused to SVD but $\mathscr{O}(10)$ new pinholes (i.e. broken decoupling capacitors between the implanted sensor strip and the APV25 preamplifier input) were found in the detector. These are not concentrated in a single location and thus do not pose an issue for SVD operation.

The radiation dose accumulated in the SVD detector was monitored by diamond radiation sensors on the beam pipe and in the detector volume [6]. Using the correlation between the diamond doses and the SVD sensor occupancies, the exposure in different layers can be estimated. During the spring 2019 run, we observed an average dose of about $8 \mathrm{krad}$ in L3 and about $2 \mathrm{krad}$ in the outer layers. The dose is higher in the backward than in the forward region and reaches up to $10 \mathrm{krad}$ in L3 mid plane.

While the SVD accumulated dose does not cause an observable effect on the physics performance at this point, we observe an increase in the L3 sensor currents to $3 \mu \mathrm{A}$ while currents are below $1 \mu \mathrm{A}$ in the outer layers. The noise increased by about $12 \%$ in the worst part of L3 while this increase is only about $5 \%$ in external layers.

The following sections present more detailed results on the SVD performance during the first Belle II physics run in spring 2019.

\section{Cluster energy and cluster signal-to-noise ratio}

A particle track traversing an SVD layer typically generates a signal over threshold on more than one strip, grouped into clusters by the reconstruction algorithm. In the following we consider SVD strip clusters associated to particle tracks to assess the SVD performance.

The energy deposited by a particle in a layer of SVD depends on the path traveled in silicon and thus on the incident angle $\alpha$ of the particle (measured relative to the normal direction on the detector surface),

$$
E \propto \frac{d}{\cos \alpha} \cdot 80 \frac{e^{-} h^{+} \text {pairs }}{\mu \mathrm{m}},
$$

where $d$ is the thickness of the sensor (300-320 $\mu \mathrm{m}$ ). The energy necessary to create one $e^{-} h^{+}$pair in silicon is $3.6 \mathrm{eV}$. The incident angle depends on the position of the sensor relative to the interaction point (IP) of the HER and LER beams. Due to these geometric considerations, we expect a higher average cluster energy in the forward than in the backward of the detector. This can be clearly observed, e.g., in the layer 3 sensors, Fig. 2.

The signal-to-noise ratio (SNR) is defined as

$$
\mathrm{SNR}=\frac{\sum_{i} S_{i}}{\sqrt{\sum_{i} N_{i}^{2}}},
$$

where $S_{i}\left(N_{i}\right)$ are the signal (noise) on strip $i$ and the summation runs over the strips included in the cluster. Very good SNR have been measured in all sensors: most probable values between 18 and 30 on the $n$-side, measuring the $z$ coordinate with short strips, and between 15 and 25 on the $p$-side, measuring the $R \phi$ coordinate with longer strips and higher associated noise. 

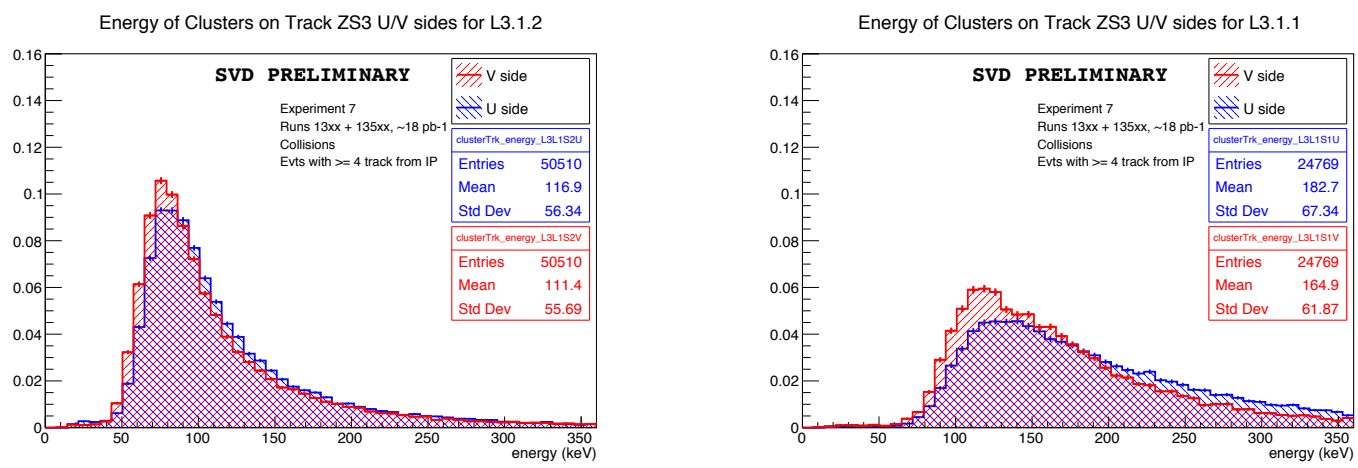

Figure 2: Cluster energy for a backward (left) and a forward (right) layer 3 sensor. The local $v(u)$ coordinate corresponds to the $n$-side ( $p$-side) of the sensor.

\begin{tabular}{|c|c|c|}
\hline \hline Efficiency & $p$-side $(R \phi)$ & $n$-side $(z)$ \\
\hline Layer 3 & $(99.75 \pm 0.02) \%$ & $(98.46 \pm 0.05) \%$ \\
Layer 4 & $(99.66 \pm 0.04) \%$ & $(99.37 \pm 0.06) \%$ \\
Layer 5 & $(99.62 \pm 0.06) \%$ & $(99.43 \pm 0.08) \%$ \\
Layer 6 & $(99.30 \pm 0.10) \%$ & $(99.30 \pm 0.10) \%$ \\
\hline \hline
\end{tabular}

Table 1: Average cluster efficiencies per SVD layer and DSSD side.

\section{Cluster efficiency}

To compute the SVD cluster efficiency, we consider reconstructed particle tracks and their intersection with the SVD layer under study. The efficiency is thus defined as the fraction of times a cluster is found within $\pm 0.5 \mathrm{~mm}$ from the intersection point. Results are shown in Table 1: On average, the efficiency is above $99 \%$ for most of the sensors, with the exception of a L3 sensor in which a read-out chip had been masked due to an unstable cable connection. Forward and backward sensors have efficiencies slightly higher than barrel sensors.

\section{Hit time resolution}

The SVD also has the capacity to determine the time of a cluster with respect to the event time. This is done by recording six subsequent flash ADC samples of the APV25 shaping curve. While no selection on the hit time is applied in the spring 2019 run, it is expected that this feature will be crucial to significantly reduce the beam background hits by rejecting off-time particles due to background events once SuperKEKB moves closer to its design luminosity.

Fig. 3 shows the SVD hit time resolution, obtained with the timing of the Central Drift Chamber (CDC) as a reference. The estimated hit time resolution is found to be 3-4 ns. 


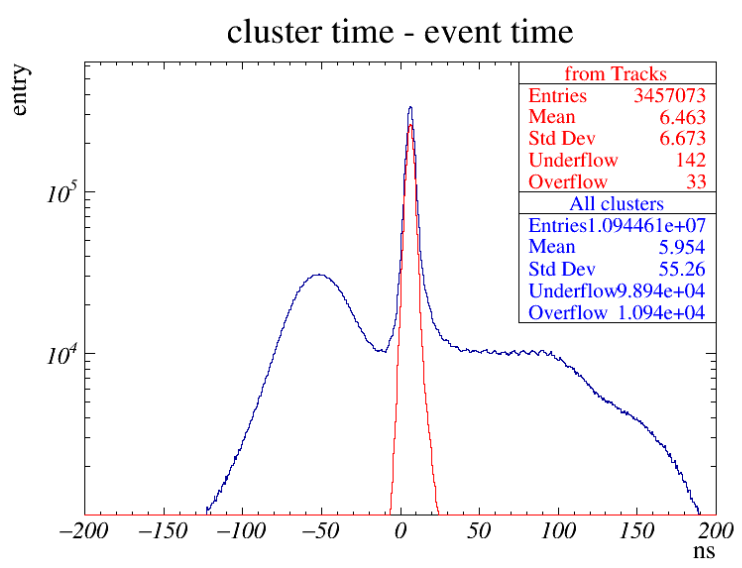

Figure 3: SVD cluster time (for all clusters and for clusters associated to reconstructed tracks) with respect to the event time (determined by the Central Drift Chamber).

\section{Summary}

After years of construction and preparation, the Silicon Vertex Detector (SVD) of the Belle II experiment was successfully completed and installed together with the pixel detector (PXD) in November 2018. From March to June 2019 it successfully performed in its first physics run: All sensors worked as expected with efficiencies around $99 \%$ and a signal-to-noise ratio between 15 and 30. The total fraction of masked strips has been below $1 \%$ and stable throughout the run. The sensor occupancy has been below $0.3 \%$ allowing track reconstruction with high efficiency. The hit time resolution shows very promising results for background rejection and tracking performance improvement, which will become crucial approaching design luminosity. No major issues were encountered in SVD during the first period of data taking.

\section{Acknowledgments}

This project has received funding from the European Union's Horizon 2020 research and innovation programme under the Marie Sklodowska-Curie grant agreements No 644294 and 822070. This work is supported by MEXT, WPI, and JSPS (Japan); ARC (Australia); BMWFW (Austria); MSMT (Czechia); AIDA-2020 (Germany); DAE and DST (India); INFN (Italy); NRF2016K1A3A7A09005605 and RSRI (Korea); and MNiSW (Poland).

\section{References}

[1] T. Abe et al. [Belle-II Collaboration], "Belle II Technical Design Report”, arXiv:1011.0352 [physics.ins-det].

[2] Y. Ohnishi et al., “Accelerator Design of SuperKEKB”, Prog. Theor. Exp. Phys. 2013 03A011 (2013).

[3] J. Brodzicka et al. [Belle Collaboration], "Physics Achievements from the Belle Experiment," PTEP 2012 (2012) 04D001 doi:10.1093/ptep/pts072 [arXiv:1212.5342 [hep-ex]]. 
[4] J. Kemmer and G. Lutz, "New Detector Concepts”, Nucl. Instrum. Meth. A 253 (1987) 365. doi:10.1016/0168-9002(87)90518-3

[5] M. J. French et al., "Design and results from the APV25, a deep sub-micron CMOS front-end chip for the CMS tracker", Nucl. Instrum. Meth. A 466 (2001) 359. doi:10.1016/S0168-9002(01)00589-7

[6] L. Vitale et al. [Belle-II SVD Collaboration], "The Monitoring System of the Belle II Vertex Detector”, PoS Vertex 2016 (2017) 051. doi:10.22323/1.287.0051 